\title{
Image Analysis of Structured Surfaces for Quantitative Topographical Characterization
}

\author{
Taylor C. Stimpson ${ }^{1}$, Devan L. Wagner ${ }^{2}$,Emily D. Cranston ${ }^{3,4}$, Jose M. Moran-Mirabal ${ }^{5 *}$ \\ ${ }^{I}$ Department of Chemical Engineering, McMaster University, Hamilton, ON, Canada \\ ${ }^{2}$ Department of Engineering Physics, McMaster University, Hamilton, ON, Canada \\ ${ }^{3}$ Department of Chemical and Biological Engineering, The University of British Columbia, , \\ Vancouver, BC, Canada \\ ${ }^{4}$ Department of Wood Science, The University of British Columbia, Vancouver, BC, Canada \\ ${ }^{5}$ Department of Chemistry and Chemical Biology, McMaster University, , Hamilton, ON, \\ Canada \\ E-mail: mirabj@mcmaster.ca
}

Keywords: Fourier transform; microfabrication; wrinkling; image filter; feature size

In the fields of functional materials, interfacial chemistry, and microscale devices, surface structuring provides an opportunity to engineer materials with unique tunable properties such as wettability, anti-fouling, crack propagation, and specific surface area. Often, the resulting properties are related to the feature sizes of the structured surfaces and therefore, it is necessary to accurately quantify these topographies. This work presents a step-by-step description of a method for the quantification of the size of periodic structures using 2D discrete Fourier Transform analysis coupled with data filtering techniques to optimize feature size extraction and reduce user bias and error. The method is validated using artificial images of periodic patterns as well as scanning electron microscopy images of gold films that are structured on different substrates. While image Fourier Transform has been used previously and is a built-in feature in some commercial and open-source image analysis software, this work details image pre-processing and feature extraction steps, and how to best apply them, which has not been described in detail elsewhere. This method can analyze engineered or natural periodic topographies (e.g., wrinkles) to enable the design of patterned materials for applications including photovoltaics, biosensors, tissue engineering, flexible electronics, and thin film metrology. 


\section{Introduction}

Surface structuring and patterning at the micro- and nanometer scale is of significant interest for applications including photovoltaics, ${ }^{[1-4]}$ biosensors, ${ }^{[4,5]}$ tissue engineering, ${ }^{[6,7]}$ flexible electronics, ${ }^{[8,9]}$ stimuli-responsive material fabrication, ${ }^{[10]}$ and thin film metrology. ${ }^{[8,11-16]}$ Structuring at this scale leads to interesting interfacial properties such as controlled wetting, tunable adhesion of cells and biomolecules, control over crack propagation in coatings, and increased specific surface area. ${ }^{[8,17,18]}$ There are many methods to produce micro- and nanoscale structured materials like photolithography, soft lithography (e.g., stamping, printing, templating, xurography), and 3D printing. ${ }^{[6,19,20]}$ Recently developed surface structuring methods take advantage of surface instabilities that arise from strain dissipation in rigid materials. Surface wrinkling is an extensively studied example of structuring based on surface instabilities, and has been used to produce functional materials with controllable properties, such as mechanical properties, ${ }^{[15,16,22,23]}$ wettability, ${ }^{[13,20]}$ conductivity, ${ }^{[4,24]}$ cell proliferation, ${ }^{[25]}$ adhesion, ${ }^{[13]}$ anti-fouling, ${ }^{[25,26]}$ stretchability, ${ }^{[13,24]}$ and optical properties. ${ }^{[15]}$ These structuring techniques make it possible to engineer materials with tunable properties, which has aided in the development of smaller, more cost effective, and higher sensitivity materials. These techniques and material properties are crucial in fabricating materials and devices for industries including renewable energy production and point of care diagnostics in healthcare.

The prevalence of structured materials in research and product development presents a need for accurate and quantifiable characterization of periodic features. Measurement of feature size is useful for understanding material production and performance, as changes in the feature sizes are often related to changes in interfacial behavior, fabrication conditions, or composition. More specifically, feature sizes in "buckled" thin films supported on flexible substrates have been used to calculate the elastic modulus, where the ability to accurately determine the feature size is critical to reliably calculate the mechanical properties. ${ }^{[16,21-23]}$ 
Image analysis using techniques like object segmentation and particle counting can be useful when examining well-defined systems, objects that are adequately separated, or features with coarse edges; however, there is a need to perform similar analysis on structures that are not easily segmented. ${ }^{[27-30]}$ Fourier Transform analysis has been previously used for determining feature sizes in periodic structures without well-defined edges that are observed in structured and patterned materials, specifically those with buckles and wrinkles. ${ }^{[3,16,31,32]}$ The method presented in this study builds on these previous developments by incorporating several filtering techniques and data fitting routines to improve the accuracy of the feature size measurement while reducing user biases.

Fourier analysis is a method that decomposes general functions into fundamental sinusoidal constituents, allowing the extraction of periodic components. ${ }^{[33,34]}$ Examples of such functions include real, time-varying signals or images, which can be analyzed to determine the temporal and spatial frequencies that they contain, respectively. For the remainder of this manuscript, "frequency" will be used to refer to the latter. In order to provide physically significant results for feature size, the analyzed function, in this case the image, must contain implicit periodicity in the features of interest.

Fourier analysis has been used in previous studies as an effective method for measuring periodic feature sizes in images of both simple and complex structured and patterned materials. In this work, we improve upon the effectiveness of Fourier analysis for the evaluation of complex surface structures (e.g., those presenting features with random orientation) by adding curve fitting and data filtering steps, as well as communicate a detailed, step-by-step guide to its implementation, which is lacking in current research literature and software. Test cases using simulated structures are used to show that features do not need to be perfectly aligned in one axis for the size information to be reliably extracted. As well, features aligned predominantly along one axis can be detected with greater intensity spectrum resolution when the power 
spectral density (PSD) is averaged over a set range of angles, rather than averaging over all angles. We also highlight that this method can effectively resolve the feature size of more complex, randomly structured surfaces such as biaxially wrinkled materials. The results support that Fourier analysis is a useful approach to quantify structured and patterned materials with periodic features, despite minor limitations, and can be employed more accurately if additional image pre-treatment and feature extraction analysis steps are included. We foresee that the presented methodology could be a useful tool to assess how feature size is related to variations in processing and material parameters and to analyze surface topographies for wide range of applications.

\section{Results \& Discussion}

\subsection{Synthetic Image Test Cases for Method Validation}

In order to validate the Fourier analysis algorithm and ensure that feature periodicity could be accurately measured, synthetic images were generated to be used as test cases. First, periodic straight black and white lines with thicknesses of 100 pixels were drawn to show that the simplest periodic structures could be detected. Following that, periodic black and white chevron lines of the same thicknesses were generated to show that the periodicity and feature width were also accurately detected when the feature angle varied over the image. Last, jigsaw puzzle-like patterns of lines with the same thickness as those used in the previous examples were created to show that even with breaks in the image data (representing pattern "disclinations"), the periodicity of the line pattern can be retrieved. This jigsaw puzzle was further fragmented to examine the effect of increasing breaks in periodicity. The jigsaw puzzle examples are especially relevant when considering the potential for the analysis of cracked samples, surfaces

with periodic domains randomly oriented, or samples with observable grain or crystal 
boundaries. The synthetic line, chevron, and jigsaw puzzle images are shown in Figure 1A, from left to right.

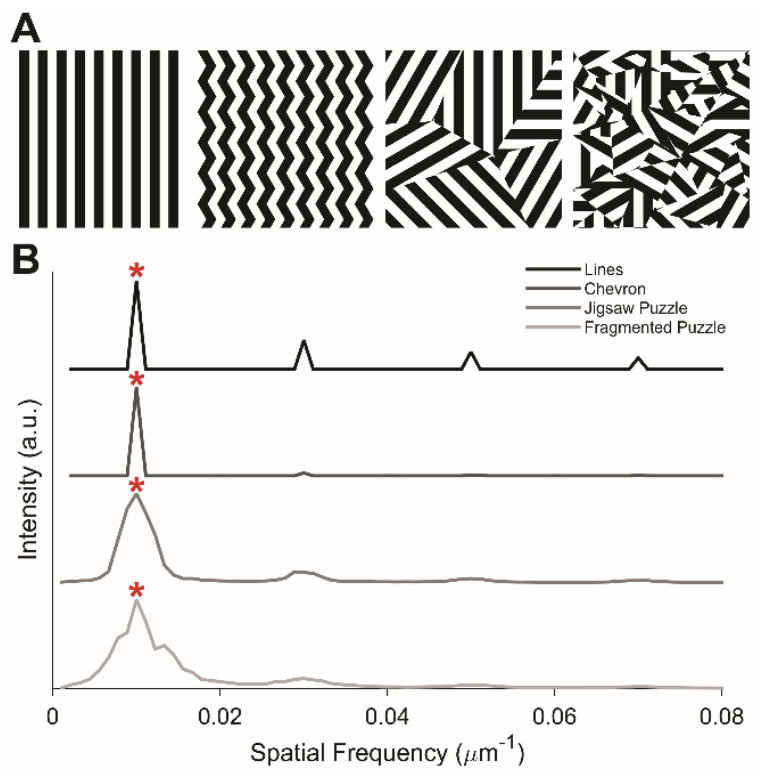

Figure 1. Fourier analysis of synthetic data test cases. Images of A) lines, chevron pattern, jigsaw puzzle, and fragmented jigsaw puzzle (from left to right) and B) the corresponding intensity spectra (from top to bottom) resulting from radially averaged PSDs over a range of 170 to $190^{\circ}$ for lines and chevron, and fully radially for the jigsaw puzzle and fragmented jigsaw puzzle. Peaks representing the spatial frequencies of interest are indicated by a red star.

Following the 2D-DFT of the images in Figure 1A, PSDs were produced and radially averaged from 170 to $190^{\circ}$ for lines and chevron patterns, and over all angles for the jigsaw puzzles, to give the intensity spectra as a function of spatial frequency (Figure 1B). Intensity spectra were cropped to spatial frequencies $<0.08 \mu \mathrm{m}^{-1}$ to highlight the relevant peaks (the full intensity spectra can be found in Figure S1 in Supporting Information). The peak with the highest intensity is the peak of interest for feature periodicity, which is the leftmost peak in these spectra as indicated in Figure 1B by the red asterisk. These peaks correspond to spatial frequencies of $0.01 \mu \mathrm{m}^{-1}$ for all four examples. Taking the inverse of these frequency values, given a scale of 1 pixel $=1 \mu \mathrm{m}$, periods of 100 pixels are obtained in a 900-pixel image basis for both images. 
These results correspond exactly to one wavelength, which in this case represents one pair of adjacent black and white lines.

It is important to note, however, that in the intensity spectrum of the jigsaw puzzle, there is a broadening of the peaks as compared to the straight line and chevron cases, despite their maximum intensities occurring at the same spatial frequency. The peak broadening in the jigsaw puzzle case occurs due to the boundaries that break the continuous periodicity, the varying orientation of the lines within the individual domains and averaging of the PSD over the full range of angles. The more irregular the periodicity of the features becomes, and the more fragmented the regions of periodic structures, the broader the peak distribution. However, as demonstrated by this example, extraction of feature size is reliant on a distinguishable peak intensity, which can still be extracted with the broader peak distribution of the jigsaw puzzle and fragmented jigsaw puzzle examples.

These three case results show that the Fourier analysis algorithm can be used to detect and calculate feature sizes for simple periodic cases. The number of periodic features and their density is linked to the peak intensity and width and is an obvious limitation of the current method as discussed further later on. Although peak broadening occurs with the introduction of randomly oriented domains, the same feature size is retrieved regardless of local variations in the feature angle or breaks in the periodicity. This is exemplified in Figure 1A, where the jigsaw puzzle has been fragmented with more boundaries (fourth image) than the original (third image). The intensity spectra shown in Figure 1B show that there is more peak broadening as the image is further fragmented, however the feature periodicity can still be identified. This becomes more important for the validity of this method as more irregularity and boundaries are introduced in the periodicity of the structures, as is often the case in real structured samples in contrast to artificially generated images. 


\subsection{Fourier Analysis of Mechanically Buckled Gold}

After validation with simple test cases, the Fourier analysis algorithm was then used to measure feature size periodicity on real structured samples. Whereas the synthetic data was composed of black and white pixels, representing the highest and lowest intensities, real images have a range of pixel intensities. This intensity range can result in broadened, less regular peaks, making the relevant data more difficult to discern from noise. To circumvent this limitation in real images, Canny edge detection was performed on the images as described in the Experimental Section.

Samples structured via mechanical buckling were analyzed to show that feature periodicity can be detected in real images of highly regular structures. A $760 \times 760$ pixel image of a gold film (45 nm thick) structured on PDMS via mechanical buckling is shown in Figure 2A, with the Canny edge detection mask of the image shown in Figure 2B. A median filter with a radius of 2 pixels was found to be the most effective to reduce speckle noise and was applied to all analyses using edge detection. A 2D-DFT was performed on the edge detection mask and the resulting PSD was averaged along one axis (angles from 170 to $190^{\circ}$ ) to obtain the intensity spectrum shown in Figure 2C. The peak corresponding to the frequency of the structures is $0.13 \mu \mathrm{m}^{-1}$ (red asterisk in Figure 2C). The inverse is taken to obtain a period of $7.6 \mu \mathrm{m}$, which is the thickness of an adjacent dark line (trough) and light line (crest). This value was validated by manually measuring the wavelength at 10 sampling locations (Table S1 of Supporting Information), to obtain a period of $7.3 \pm 0.3 \mu \mathrm{m}$. The peak with the second highest intensity, at $0.41 \mu \mathrm{m}^{-1}$, corresponds to $2.5 \mu \mathrm{m}$, which is the thickness of the light lines in Figure 2A, or the peaks of the sinusoidal wave patterns. 


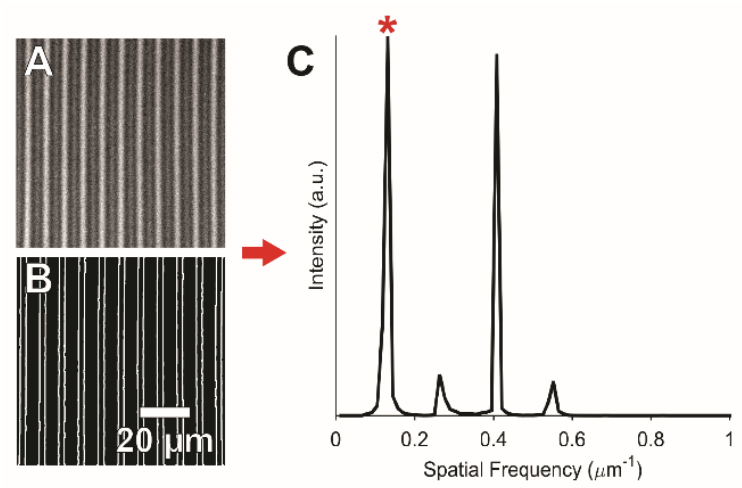

Figure 2. Fourier analysis of mechanically buckled gold films (45 nm-thick) on PDMS. A) SEM image of buckled gold and B) the corresponding Canny edge detection masks. Fourier analysis is performed on the edge detection mask to produce a PSD that is radially averaged over a range of 170 to $190^{\circ}$, giving the C) intensity spectra for the sample. The peak representing the spatial frequency of interest is indicated by a red star.

\subsection{Fourier Analysis of Thermally Structured Gold}

Finally, Fourier analysis was performed on $100 \mathrm{~nm}$-thick gold films deposited on polystyrene substrates and structured through biaxial and uniaxial thermal shrinking. Images of both biaxially and uniaxially structured gold are shown in Figure 3A, with their respective edge detection masks shown in Figure 3B. These structured samples demonstrate that feature periodicity can be measured in more complex structures. The uniaxially structured samples are more regular, demonstrating that PSD averaging within a confined range of angles is still effective for these more complex features. Biaxially structured samples represent the highest complexity of structured surfaces analyzed in this work, as they are the most irregular and randomly oriented. However, these features still present repeating features (e.g., wrinkle width) and thus Fourier analysis can still be used. 


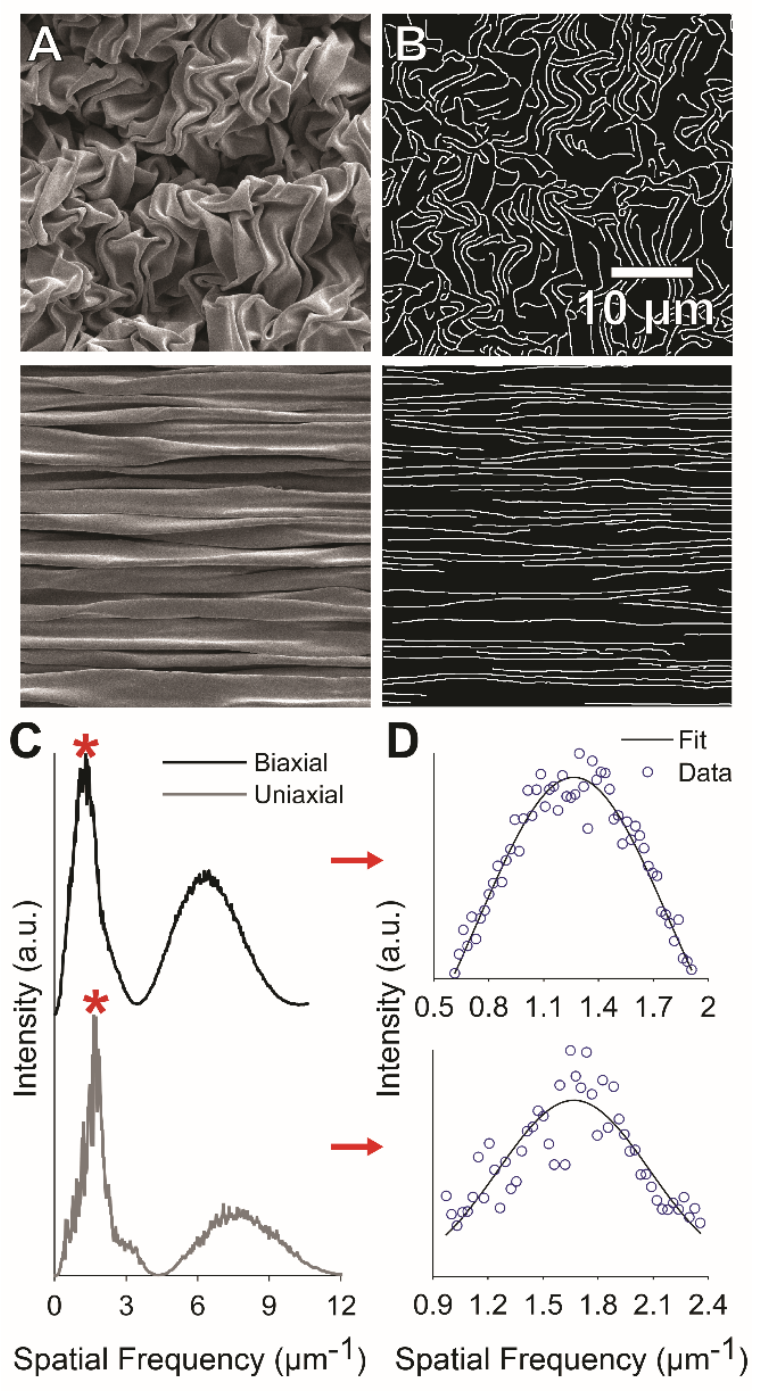

Figure 3. Fourier analysis of biaxial and uniaxial thermal structuring of $100 \mathrm{~nm}$ thick gold films on polystyrene using A) SEM images of biaxial (top) and uniaxial (bottom) thermally structured gold and B) the corresponding Canny edge detection masks. This yields C) the intensity spectra for both samples. The peak of interest is indicated by a red star. These peaks are D) fit to a Gaussian for biaxial (top) and uniaxial (bottom) structured samples, over the spatial frequency range shown in the plots.

Fourier transforms were performed on the edge detection masks. The resulting PSD from the 2D-DFT was averaged over the full range of angles (biaxially structured films) and from a range of 70 to $110^{\circ}$ (uniaxially structured films) to obtain the intensity spectra in Figure 3C. The peak of interest for the feature periodicity, or characteristic frequency, of each sample, is indicated 
by the red asterisk in Figure 3C. The second, less intense peak for each case corresponds to single wrinkles that are transformed into double line segments following edge detection. This was confirmed through visual inspection and manual length measurement. Since the data in both thermal structuring cases are more irregular and have less ideally periodic structures compared to the sinusoidal wave patterns (Figure 2) and synthetic images (Figure 1), the intensity spectrum peaks have a larger spread. Thus, the peaks of interest were fit to a Gaussian curve as discussed in the Experimental Section.

The curve fits were performed over the spatial frequency ranges shown in Figure 3D, for biaxially (top) and uniaxially (bottom) structured gold films. From the Gaussian fits (Equation 4) $\bar{k}$ is the spatial frequency of interest that gives the reciprocal of the relevant feature wavelength. For biaxially and uniaxially structured gold, $\bar{k}$ was 1.26 and $1.65 \mu \mathrm{m}^{-1}$, which translates into feature periodicities of $0.8 \pm 0.2$ and $0.6 \pm 0.1 \mu \mathrm{m}$, respectively. These values were validated by measuring the characteristic wavelength manually using the original images at 10 sampling locations, to obtain $0.8 \pm 0.2$ and $0.6 \pm 0.2 \mu \mathrm{m}$, for biaxial and uniaxial structures, respectively. These manually measured values are provided in Table S1 of Supporting Information.

The broad peaks in the intensity spectra exhibited by the biaxially and uniaxially structured materials arise from the irregularity and distribution in wrinkle size across the sample, similar to what occurred in the jigsaw puzzle test case. Although there is a characteristic feature size that can be extracted from the spatial frequency at the peak intensity of the spectra, the broad distribution arises from polydispersity in the wrinkle widths. This method uses the mean of the Gaussian curve fit of the peak of interest, so there is the inherent assumption that even with a broad peak distribution, the mean represents the characteristic and most dominant feature size. This represents a limitation, whereby too broad of a peak would render the mean of the Gaussian an ineffective representation of the dominant feature sizes. However, we make the overall 
assumption in using this method that there exists one dominant feature size in which case the peak should be sufficiently narrow for accurate feature size extraction.

We have shown with this work that using Fourier analysis, feature sizes can be successfully extracted from synthetic image test cases, as well as the highly periodic mechanically buckled and more irregularly structured biaxial and uniaxial thermally structured gold films. These cases show that Fourier analysis is a viable method for measuring feature size for structured and patterned materials that have periodically occurring features. We have also addressed that although irregularity in feature size as well as boundaries that break periodicity exist, especially in real structured and patterned materials, and that this causes peak broadening, this method is still effective for the extraction of the underlying and most frequently occurring feature size. While Fourier Transform analysis has previously been employed for topographical characterization of structured surfaces, the algorithm presented in this work introduces an image pre-processing step and a PSD peak fitting step, which combined lead to improved accuracy in the extracted feature size and periodicities.

The determination of feature periodicity through Fourier analysis is a useful characterization tool whereby changes in feature size can be related to variations in material properties, fabrication, or composition. For example, if the gold sputtered film used here was not uniform in thickness, or if there were other contaminant metals, then the structures would be different sizes and non-uniform across the film surface (because the feature size is proportional to the film mechanics and a change in thickness/composition affects this). This work also serves as a useful guide for the application of this analysis method for materials scientists and engineers to accurately quantify and characterize feature sizes of structured and patterned materials. The application of Fourier analysis for periodic feature size determination could be used to quantify how changes in material preparation parameters affect resulting properties including but not limited to elastic modulus, hydrophobicity or hydrophilicity, conductivity, adhesion, fouling, 
stretchability, and optical properties. Specifically, accurate characterization of periodic feature size could be applied to many areas of study including the extraction of mechanical moduli from buckled films; ${ }^{[15,16,22,23]}$ assessment of how drying or crosslinking parameters affect the topography of wrinkled hydrogel systems; ${ }^{[43]}$ analysis of cell growth, proliferation, and alignment as a function of surface topography; ${ }^{[44,45]}$ quantification of the effect of structure size on anti-fouling properties; ${ }^{[26]}$ and the characterization of current and resistance of stretchable electronics as a function of wrinkle size. ${ }^{[24]}$

\subsection{Implications \& Limitations of Fourier Analysis for Feature Periodicity and Size Determination}

We have shown that the analysis method presented is robust, straightforward in its use, and streamlines the analysis of structured systems such as those discussed above. In addition, as seen in Figure 3C and 3D, Fourier analysis can be used to extract the statistical distributions that govern feature sizes in materials structured via surface instabilities. However, due to the inherent properties of Fourier transformations, there are limitations because of the information density present in real images.

Information density in the context of Fourier analysis refers to the number of repeats of the periodic features present in a single image as well as how closely packed they are from one another. There are two distinct limitations in this regard. First, and more inherent to the mathematical definition of the Fourier transform, the magnification and field of view of the image must be such to capture enough periodic features. This is due to the prerequisite of implicit periodicity in the features of interest discussed in the Introduction. Second, there is a trade-off between the number of features captured in a single image and the ease of detecting or distinguishing their edges. 
To demonstrate this, recall the definition of frequency dynamic range $n / 2$ and frequency resolution $(1 / 2)\left(1 / D_{n}\right)$ as presented in the Experimental Section. Assuming a constant image size (and a constant dynamic range), an image with an insufficient number of features within a field of view ( $\mathrm{a} \mathrm{D}_{\mathrm{n}}$ which is too small for a given feature size) would cause the peak of interest in the PSD to be shifted towards the origin of frequency space. This, compounded with the sources of noise discussed above, leads to a characteristic peak that is indistinguishable from the background noise signal. Conversely, a large field of view (large $\mathrm{D}_{\mathrm{n}}$ ) would cause the peak of interest to shift away from the origin and occupy more of the dynamic range. Although this is the more ideal case, limitations in edge detection algorithms would consequently make it difficult to distinguish highly dense features.

Both limitations in information density are further exacerbated due to practical limitations in sample imaging (SEM or related modalities), where it may be difficult to acquire data with an ideal field of view and/or magnification. Overall, this means that the experimentalist must exhibit some degree of a priori knowledge of their system in order to select a dynamic range and field of view that adequately frames the peak of interest within the frequency space.

Future work with the Fourier analysis method could focus on various improvements to overcome the aforementioned limitations. Focus can be placed on the investigation of additional filtering techniques and refinement to enable the consistent extraction of relevant information without introducing user bias from the inputs at these steps. Additionally, machine learning models such as convolutional neural network-based regression could be used to automatically analyze systems such as those presented above, therefore drastically reducing the need for $a$ priori knowledge. The large dataset of previously characterized images required to train such a model could therefore be built using our Fourier analysis method due to the efficiency improvements over manual feature detection. 


\section{Conclusions}

Fourier analysis has been applied as a method for characterizing feature sizes of periodic structures and patterns, as shown with examples of synthetic images and images of wrinkled gold films on compliant substrates. We have shown that the use of Fourier analysis in conjunction with filtering and data fitting techniques gives reliable and accurate measurements of feature sizes of periodic structures for all cases tested. Most notably, if properly employed, Fourier analysis can be effective for extracting accurate feature sizes of even highly irregular and complex structures, as demonstrated with biaxial thermally structured gold. Manually measuring the feature sizes of structures with such irregularity is tedious, inefficient, and can lead to large error ranges in the measured value. Thus, reliable Fourier analysis is especially valuable for measuring feature sizes of these types of irregularly structured materials.

The application of Fourier analysis to feature size detection is limited, however, by information density within the images used for analysis. These limitations are exacerbated by imaging instrumentation resolution and sample preparation requirements. In future iterations of this method, focus could be placed on the investigation of additional filtering techniques and on reducing user bias and required a priori knowledge of the system for feature size characterization.

This work shows a few examples of different types of structured surfaces and feature morphologies that can be analyzed with Fourier analysis, and gives an overview of how this might be used for other structured and patterned surfaces for topographical characterization. In general, this analysis method proves useful in quantifying feature size for the purpose of relating observed and measured material properties to feature size. This is especially relevant for optimizing material fabrication for different applications and developing materials with tunable surface properties. Although Fourier analysis is a well-known method for image analysis, the 
algorithm and specific methods presented will aid in the accessibility and accuracy of the method for a wider range of studies. We envision that this method will be applicable in quantifying feature sizes for material characterization in many research areas. These areas include stretchable electronics, structured electrodes for point of care diagnostics, structured hydrogels, nanowire arrays, measurement of thin film elastic moduli through strain induced mechanical buckling, and many more emerging areas in structured and patterned materials on the micro- and nanoscales.

\section{Experimental}

\subsection{Polystyrene Substrate Preparation}

Pre-stressed polystyrene shrink films (Graphix Shrink Film, Maple Heights, OH, USA) were used as substrates for the deposition of thin gold films for thermal structuring methods (i.e., to prepare structured samples for image analysis). The substrates were cut into $2 \times 2 \mathrm{~cm}$ squares and $2 \times 5 \mathrm{~cm}$ rectangles for biaxial and uniaxial thermal structuring, respectively. The polystyrene was cut with a RoboPro CE5000-40-CRP cutter (Graphtec America Inc., Irvine, CA, USA) with a CB15UB ceramic blade using parameters of 30,1 , and 1 , for force, quality, and speed, respectively. The cut substrates were cleaned in consecutive baths of isopropanol, ethanol, and ultrapure water (Milli-Q A10 Purification System, Millipore, Etobicoke, CA) for 5 minutes each under $50 \mathrm{rpm}$ of orbital agitation. The substrates were then dried with compressed nitrogen and stored in sealed containers on the benchtop until further use.

\subsection{Polydimethylsiloxane (PDMS) Substrate Preparation}

PDMS (SYLGARD® 184 Silicone Elastomer Kit, Dow Corning Corporation, Midland, MI, USA) substrates were prepared using a 10:1 mass ratio of elastomer base to curing agent. The base and curing agent were mixed for three minutes using a stirring rod. The mixture was 
degassed using a vacuum pump for 30 minutes, then immediately poured into a rectangular mold of $6 \times 1 \times 2 \mathrm{~cm}(1 \times \mathrm{w} \times \mathrm{h})$. The elastomer substrates were left to cure at room temperature for 48 hours before removing them from the mold for use.

\subsection{Gold Film Deposition}

Gold (99.999\% purity, LTS Chemical Inc., Chestnut Ridge, NY, USA) films of $100 \mathrm{~nm}$ thickness were deposited onto polystyrene substrates using a Torr Compact Research Coater CRC-600 manual planar magnetron sputtering system (Torr International, New Windsor, NY, USA) using a deposition rate of $0.5 \AA$ /s. Gold films were deposited onto PDMS substrates via an Edwards Sputter Coater S150B (Edwards, Crawly, UK) using an argon gas plasma chamber to obtain a thickness of $\sim 45 \mathrm{~nm}$ (gold deposition rate is rated at $15 \mathrm{~nm} \mathrm{~min}^{-1}$, and sputtering was performed for 3 minutes).

\subsection{Gold Film Structuring}

Gold films were structured using three different approaches to highlight the range of features that can be resolved by the Fourier analysis method described in this work. Gold films were deposited onto $2 \times 2 \mathrm{~cm}$ and $2 \times 5 \mathrm{~cm}$ polystyrene substrates at $100 \mathrm{~nm}$ thicknesses, and onto 6 $\times 1 \times 2 \mathrm{~cm}(1 \times \mathrm{w} \times \mathrm{h})$ PDMS substrates at a thickness of $45 \mathrm{~nm}$. Gold films on polystyrene were thermally structured, by heating the sample to $135^{\circ} \mathrm{C}$, above the glass transition temperature of polystyrene, which caused the substrates to shrink. Because of the elastic modulus mismatch between the thin film and substrate, as the pre-stressed polystyrene shrinks the gold film buckles, resulting in a wrinkled morphology. Thermal structuring can be performed on samples which are unconstrained (biaxial structuring) or constrained along one axis via clamps (uniaxial structuring), only allowing wrinkling to occur perpendicular to the clamping axis. ${ }^{[35]}$ PDMS was placed between the sample and the metal clamp for uniaxial structuring to ensure that the sample remained securely clamped (avoiding slippage) during 
shrinking. The biaxial and uniaxial thermal structuring methods are demonstrated in the schematic representations shown in Figure 4A and 4B, respectively.

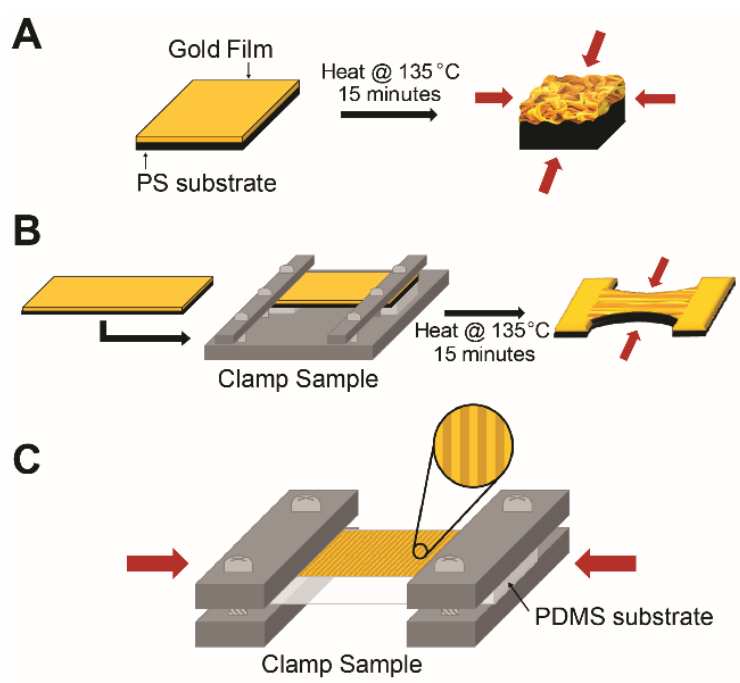

Figure 4. Schematic representations of gold film structuring through A) biaxial thermal structuring, B) uniaxial thermal structuring, and C) mechanical buckling methods.

The other structuring approach, called mechanical buckling in this work, uses PDMS as a compliant elastomeric substrate for mechanical compression and buckling of a gold film based on the strain-induced elastic buckling instability for mechanical measurements method described by Stafford et al. ${ }^{[15]}$ Buckling was induced using an in situ tensile device (SEMTester DAQ 8000-0014, MTI Instruments Inc., Albany, NY, USA) that couples to a JEOL 6610LV (JEOL, Tokyo, Japan) scanning electron microscope (SEM). Compression, or strain, was applied to the sample while inside the SEM through software (MTESTQuattro-MaterialsTesting-System by ADMET Incorporated, V3-07-01, Norwood, MA, USA) that interfaces with the tensile device. Strains between 0.5 and $1 \%$ were applied to the sample to compress the substrate sufficiently to cause the gold film to buckle into a sinusoidal wave pattern. A schematic representation of the mechanical buckling method is shown in Figure 4C. 


\subsection{Scanning Electron Microscopy (SEM)}

Thermally structured gold films on polystyrene were prepared for SEM imaging by mounting the samples onto 1" stainless steel stubs using carbon tape and nickel paint to establish contact between the stub and sample and reduce charging during imaging. Uniaxially structured samples were cut to size at the ends to fit within the stub dimensions, ensuring that the center of the sample remained intact. Images were taken using a JEOL 7000F SEM (JEOL, Tokyo, Japan) at acceleration voltages of 1.5 or $2 \mathrm{kV}$ and working distances between 3 and $6 \mathrm{~mm}$.

Mechanically buckled gold films on PDMS were imaged using a JEOL 6610LV (JEOL, Tokyo, Japan). Images were taken while the samples were buckled using an in situ tensile device inside the SEM. No conductive coating or additional sample preparation was required to obtain the SEM images. Images were taken using an acceleration voltage of $2 \mathrm{kV}$ and a working distance of $25 \mathrm{~mm}$.

\subsection{Fourier Transforms}

The Fourier transform of an image is defined by the two-dimensional discrete Fourier transform (2D-DFT). This treatment considers the discrete nature of the pixels which make up the image space, as opposed to data points in a continuous, closed-form signal. The 2D-DFT, F( $\left.\mathrm{k}_{\mathrm{x}}, \mathrm{k}_{\mathrm{y}}\right)$ of a square $\mathrm{n} \times \mathrm{n}$ image $\mathrm{f}(\mathrm{x}, \mathrm{y})$, is given by:

$F\left(k_{X}, k_{y}\right) \equiv \sum_{x=0}^{n-1} \sum_{y=0}^{n-1} f(x, y) e^{-\frac{2 \pi i}{n}\left(k_{x} x+k_{y} y\right)}$

where $\mathrm{x}$ and $\mathrm{y}$ are the pixel coordinates in image space, $\mathrm{k}_{\mathrm{x}}$ and $\mathrm{k}_{\mathrm{y}}$ are the reciprocal pair of coordinates in frequency space, and $i$ is the imaginary number. It should be noted that it is a common convention for the resulting 2D-DFT to be shifted such that the origin, or a frequency of 0 , is at the center of the frequency space as opposed to the upper left corner as is the case in 
image space. Therefore, an $n \times n$ image that has a real side length of $D_{n}$ will have a usable frequency range from $(-\sqrt{2} / 2)\left(n / D_{n}\right)$ to $(+\sqrt{ } 2 / 2)\left(n / D_{n}\right)$.

In order to extract information regarding the periodicity of structures (such as wrinkles and arrays of features) from an image, the power spectral density (PSD) must be examined. The PSD of an image or signal represents the power content of each frequency. This spectrum is a real-valued function which contains physical information regarding the strength of any given signal as a function of frequency. The PSD, $\mathrm{P}\left(\mathrm{k}_{\mathrm{x}}, \mathrm{k}_{\mathrm{y}}\right)$, of an image is given by the magnitude of the DFT, calculated as the product of the transform $\mathrm{F}\left(\mathrm{k}_{\mathrm{x}}, \mathrm{k}_{\mathrm{y}}\right)$ by its complex conjugate $\mathrm{F}^{*}\left(\mathrm{k}_{\mathrm{x}}, \mathrm{k}_{\mathrm{y}}\right)$ in frequency space. Peaks or areas of high intensity in the PSD can be interpreted in polar coordinates such that the radius and angle correspond to the frequency and direction of periodic features existing in image space, respectively.

\subsection{Digital Image Processing \& Analysis}

Images from SEM as well as images generated from synthetic data were used for digital image processing and data analysis. Images taken at different magnifications were cropped using ImageJ $1.52 \mathrm{a}^{[36]}$ (National Institutes of Health, US) to $900 \times 900$ pixels or $760 \times 760$ pixels for thermal structuring and mechanical buckling, respectively. The images were also adjusted for contrast and brightness in ImageJ to improve feature detection. Synthetic images of $900 \times 900$ pixels were generated using Adobe Illustrator (Adobe, San Jose, CA, US) to use as test cases for the accuracy and robustness of the Fourier analysis algorithm.

Canny edge detection was performed using ImageJ to detect "edges" or areas that exhibit sharp spatial intensity gradients in the image. ${ }^{[37]}$ However, to remove false edges and reduce additional spurious, high-frequency noise from over-thresholding in the edge detection, median filtering was performed on the edge masks in MATLAB (MATLAB R2014b, MathWorks, 
US) ${ }^{[38]}$ A filtering window of 2 pixels was found to be most effective at removing individual speckle pixels while preserving edges.

Fourier transform analyses were then performed on the filtered edge masks using an implementation of the Fast Fourier Transform. ${ }^{[39]}$ This and all subsequent steps were also performed in MATLAB using a graphical user interface. The full MATLAB script and stepby-step guide to using the graphical user interface (Figure S2 - S7) are included in Supporting Information. The PSD was then calculated and averaged across all angles specified, around the origin of the frequency space, to obtain a 1D intensity spectrum. The angular averaging was done for all radii, $\mathrm{k}_{\mathrm{r}}$, from $k_{r} \equiv \sqrt{k_{x}^{2}+k_{y}^{2}}=\left|\frac{1}{2} \frac{1}{D_{n}}\right|$ to $\left|\frac{1}{2} \frac{n}{D_{n}}\right|$. Although the origin of the frequency space is located at the center of the image, the slight offset in $\mathrm{k}_{\mathrm{r}}$ was introduced to account for the even number of pixels in the side length of the images. The dynamic range in frequency space, or the ratio of maximum to minimum resolvable frequency, is therefore given by $\mathrm{n} / 2$. In addition, the averaging was done for all angles from 0 to $360^{\circ}$ or within a specified range for randomly oriented structures or those aligned along a single axis, respectively.

The resulting intensity spectra were then filtered to reduce noise and artifacts. This allowed the straightforward extraction of the frequencies that corresponded to the feature sizes of interest. A common artifact in many physical systems, known as "pink noise" or " $1 /$ f noise," manifests as an increased background signal in PSDs that is proportional to $1 / \mathrm{k}_{\mathrm{r}}{ }^{[40]}$ This noise was reduced by dividing the intensity spectrum by an artificial signal that followed this same characteristic form across the appropriate frequency range mentioned above.

Lastly, Gaussian and inverse Gaussian windows were used as multiplicative low- and high-pass filters, respectively. The high-pass filter was used to remove the background (mean-level) component of each image while the low-pass filter was used to lessen the amplification of high frequency components that occurs following edge detection. The tapering nature of the 
Gaussian curve was used to mitigate discontinuities and additional artifacts in the resulting intensity spectra. The low-pass and high-pass filter windows, $\mathrm{G}_{\mathrm{L}}$ and $\mathrm{G}_{\mathrm{H}}$, respectively, of independent size d were defined by Equation 2 and Equation 3, respectively.

$G_{L}=e^{-\frac{k_{r}^{2}}{4 d^{2}}}$

$G_{H}=1-e^{-\frac{k_{r}^{2}}{4 d^{2}}}$

For all cases, a window size of $d=0$ to 10 pixels was used. Within frequency space, this corresponds to a bandwidth of $\left(d_{n} / 2\right)\left(1 / D_{n}\right)$.

For thermally structured (and thus more irregular) samples, the characteristic feature size was extracted from fully filtered intensity spectra through a curve fitting routine based on the Nelder-Mead simplex minimization algorithm. ${ }^{[41]}$ This routine determines the optimum fitting parameters required to match the intensity spectra data to a closed-form function by following a least-squares approach. In this work, the closed-form function was taken to be a Gaussian curve of the form:

$g=\frac{A}{\sigma \sqrt{2 \pi}} e^{-\frac{1}{2}\left(\frac{k_{r}-\bar{k}}{\sigma}\right)^{2}}$

Where the fitting parameters are the spatial frequency of the characteristic feature, $\overline{\mathrm{k}}$, the standard deviation of the distribution, $\sigma$, and a scaling factor, A. A Gaussian function was used to capture the statistical distribution of feature sizes in wrinkled structures. Gaussian distributions were used because, unlike other distributions such as the Cauchy distribution, ${ }^{[42]}$ they have a mathematically defined mean which is the parameter of interest in this analysis method. In the case of synthetic images and samples prepared via the mechanical buckling method, the resulting intensity spectra exhibited sharp peaks and could therefore be 
characterized by the first (fundamental) peak or extracted via visual inspection. Mathematically, these sharp peaks are defined by the Dirac-delta, or the limit of Equation 4 as $\sigma \rightarrow 0$. Because the intensity spectra are functions in reciprocal space, the characteristic feature size was calculated by taking the inverse of $\overline{\mathrm{k}}$.

\section{Acknowledgements}

The authors thank C. Butcher of the Canadian Centre for Electron Microscopy (CCEM) for his expertise and assistance with SEM data acquisition of mechanically buckled films, D. Osorio for SEM of thermally structured films, and S. Saem for sputtering of gold films. J.M.M. and E.D.C. are recipients of Early Researcher awards from the Ontario Ministry of Research and Innovation. J.M.M. is the Tier 2 Canada Research Chair in Micro and Nanostructured Materials. T.C.S is the recipient of a Queen Elizabeth II Ontario Graduate Scholarship. D.L.W. is the recipient of the Alexander Graham Bell Canada Graduate Scholarship. The research presented here was supported by funding through the Natural Sciences and Engineering Research Council and made use of facilities at the CCEM and the McMaster Centre for Emerging Device Technologies (CEDT). Funding was provided through the Natural Sciences and Engineering Research Council of Canada (Discovery Grants RGPIN 2019-06433 and 05252). The manuscript was written through contributions of all authors. All authors have given approval to the final version of the manuscript. 


\section{References}

[1] Zhang, B.; Zhang, M.; Song, K.; Li, Q.; Cui, T. Shrink Induced Nanostructures for Energy Conversion Efficiency Enhancement in Photovoltaic Devices. Appl. Phys. Lett. 2013, 103 (2). https://doi.org/10.1063/1.4812184.

[2] Kim, J. B.; Kim, P.; Ṕgard, N. C.; Oh, S. J.; Kagan, C. R.; Fleischer, J. W.; Stone, H. A.; Loo, Y. L. Wrinkles and Deep Folds as Photonic Structures in Photovoltaics. Nat. Photonics 2012, 6 (5), 327-332. https://doi.org/10.1038/nphoton.2012.70.

[3] Chen, Z.; Young Kim, Y.; Krishnaswamy, S. Anisotropic Wrinkle Formation on Shape Memory Polymer Substrates. J. Appl. Phys. 2012, 112 (12). https://doi.org/10.1063/1.4770483.

[4] Gabardo, C. M.; Zhu, Y.; Soleymani, L.; Moran-Mirabal, J. M. Bench-Top Fabrication of Hierarchically Structured High-Surface-Area Electrodes. Adv. Funct. Mater. 2013, 23 (24), 3030-3039. https://doi.org/10.1002/adfm.201203220.

[5] Kim, H. S.; Crosby, A. J. Solvent-Responsive Surface via Wrinkling Instability. Adv. Mater. 2011, 23 (36), 4188-4192. https://doi.org/10.1002/adma.201101477.

[6] Jeon, H. J.; Simon, C. G.; Kim, G. H. A Mini-Review: Cell Response to Microscale, Nanoscale, and Hierarchical Patterning of Surface Structure. J. Biomed. Mater. Res. Part B Appl. Biomater. 2014, 102 (7), 1580-1594.

https://doi.org/10.1002/jbm.b.33158.

[7] Gao, L.; Ma, L.; Yin, X. hong; Luo, Y. chen; Yang, H. yong; Zhang, B. Nano- and Microfabrication for Engineering Native-Like Muscle Tissues. Small Methods 2020, 4, 1-13. https://doi.org/10.1002/smtd.201900669.

[8] Mechael, S. S.; Wu, Y.; Schlingman, K.; Carmichael, T. B. Stretchable Metal Films. Flex. Print. Electron. 2018, 3, 1-24.

[9] Ling, H.; Liu, S.; Zheng, Z.; Yan, F. Organic Flexible Electronics. Small Methods 2018, 2, 1-33. https://doi.org/10.1002/smtd.201800070. 
[10] Villares, A.; Moreau, C.; Dammak, A.; Capron, I.; Cathala, B. Kinetic Aspects of the Adsorption of Xyloglucan onto Cellulose Nanocrystals: Supporting Information. Soft Matter 2015, 1-4.

[11] Freschauf, L. R.; McLane, J.; Sharma, H.; Khine, M. Shrink-Induced Superhydrophobic and Antibacterial Surfaces in Consumer Plastics. PLoS One 2012, 7 (8), 1-7. https://doi.org/10.1371/journal.pone.0040987.

[12] Sharma, H.; Digman, M. A.; Felsinger, N.; Gratton, E.; Khine, M. Enhanced Emission of Fluorophores on Shrink-Induced Wrinkled Composite Structures. Opt. Mater. Express 2014, 4 (4), 753. https://doi.org/10.1364/ome.4.000753.

[13] Rodríguez-Hernández, J. Wrinkled Interfaces: Taking Advantage of Surface Instabilities to Pattern Polymer Surfaces. Prog. Polym. Sci. 2015, 42, 1-41. https://doi.org/10.1016/j.progpolymsci.2014.07.008.

[14] Li, B.; Cao, Y. P.; Feng, X. Q.; Gao, H. Mechanics of Morphological Instabilities and Surface Wrinkling in Soft Materials: A Review. Soft Matter 2012, 8 (21), 5728-5745. https://doi.org/10.1039/c2sm00011c.

[15] Stafford, C. M.; Harrison, C.; Beers, K. L.; Karim, A.; Amis, E. J.; Vanlandingham, M. R.; Kim, H. C.; Volksen, W.; Miller, R. D.; Simonyi, E. E. A Buckling-Based Metrology for Measuring the Elastic Moduli of Polymeric Thin Films. Nat. Mater. 2004, 3 (8), 545-550. https://doi.org/10.1038/nmat1175.

[16] Gill, U.; Sutherland, T.; Himbert, S.; Zhu, Y.; Rheinstädter, M. C.; Cranston, E. D.; Moran-Mirabal, J. M. Beyond Buckling: Humidity-Independent Measurement of the Mechanical Properties of Green Nanobiocomposite Films. Nanoscale 2017, 9 (23), 7781-7790. https://doi.org/10.1039/C7NR00251C.

[17] Li, S. J.; Wu, K.; Yuan, H. Z.; Zhang, J. Y.; Liu, G.; Sun, J. Formation of Wrinkled Patterns in Metal Films Deposited on Elastic Substrates: Tunability and Wettability. Surf. Coatings Technol. 2019, 362 (November 2018), 35-43. 
https://doi.org/10.1016/j.surfcoat.2019.01.088.

[18] Prathapan, R.; Berry, J. D.; Fery, A.; Garnier, G.; Tabor, R. F. Decreasing the Wettability of Cellulose Nanocrystal Surfaces Using Wrinkle-Based Alignment. ACS Appl. Mater. Interfaces 2017, 9 (17), 15202-15211. https://doi.org/10.1021/acsami.7b03094.

[19] Chiche, A.; Stafford, C. M.; Cabral, J. T. Complex Micropatterning of Periodic Structures on Elastomeric Surfaces. Soft Matter 2008, 4 (12), 2360-2364. https://doi.org/10.1039/b811817e.

[20] Xia, Y.; Qin, D.; Yin, Y. Surface Patterning and Its Application in Wetting/Dewetting Studies. Curr. Opin. Colloid Interface Sci. 2001, 6 (1), 54-64. https://doi.org/10.1016/S1359-0294(00)00088-1.

[21] Wilder, E. A.; Guo, S.; Lin-Gibson, S.; Fasolka, M. J.; Stafford, C. M. Measuring the Modulus of Soft Polymer Networks via a Buckling-Based Metrology. Macromolecules 2006, 39 (12), 4138-4143. https://doi.org/10.1021/ma060266b.

[22] Cranston, E. D.; Eita, M.; Johansson, E.; Netrval, J.; Salajkov, M.; Arwin, H.; Lars, W. Determination of Young's Modulus for Nanofibrillated Cellulose Multilayer Thin Films Using Buckling Mechanics. Biomacromolecules 2011, 12, 961-969. https://doi.org/10.1021/bm101330w.

[23] Hahm, S.-W.; Hwang, H.-S.; Kim, D.; Khang, D.-Y. Buckling-Based Measurements of Mechanical Moduli of Thin Films. Electron. Mater. Lett. 2009, 5 (4), 157-168. https://doi.org/10.3365/eml.2009.12.157.

[24] Khang, D. Y.; Rogers, J. A.; Lee, H. H. Mechanical Buckling: Mechanics, Metrology, and Stretchable Electronics. Adv. Funct. Mater. 2009, 19 (10), 1526-1536. https://doi.org/10.1002/adfm.200801065.

[25] Rodr, J. Wrinkled Polymer Surfaces; 2019. https://doi.org/10.1007/978-3-030-05123-5.

[26] González-Henríquez, C. M.; Sarabia-Vallejos, M. A.; Terraza, C. A.; del Campo- 
García, A.; Lopez-Martinez, E.; Cortajarena, A. L.; Casado-Losada, I.; MartínezCampos, E.; Rodríguez-Hernández, J. Design and Fabrication of Biocompatible Wrinkled Hydrogel Films with Selective Antibiofouling Properties. Materials Science and Engineering C. 2019, pp 803-812. https://doi.org/10.1016/j.msec.2018.12.061.

[27] Pelz, J. B. Morphological Image Segmentation by Local Granulometric Size Distributions. J. Electron. Imaging 2006, 1 (1), 46. https://doi.org/10.1117/12.55174.

[28] Gazeta, F. N. Particle Size Distribution Analysis of Course Aggregate Using Digital Image Processing. 1998, 28 (6), 921-932.

[29] Vaithiyanathan, V.; Rajappa, U. A Review on Clustering Techniques in Image Segmentation. Int. J. Appl. Eng. Res. 2013, 8 (20 SPEC. ISSUE), 2685-2688.

[30] Venkataraman, S.; Allison, D. P.; Qi, H.; Morrell-Falvey, J. L.; Kallewaard, N. L.; Crowe, J. E.; Doktycz, M. J. Automated Image Analysis of Atomic Force Microscopy Images of Rotavirus Particles. Ultramicroscopy 2006, 106 (8-9), 829-837. https://doi.org/10.1016/j.ultramic.2006.01.014.

[31] Na, Y.; Pourdeyhimi, B. Assessing Wrinkling Using Image Analysis and Replicate Standards. Text. Res. 1995, 65 (3), 149-157.

[32] Krill, C. E.; Birringer, R. Estimating Grain-Size Distributions in Nanocrystalline Materials from X-Ray Diffraction Profile Analysis. Philos. Mag. A Phys. Condens. Matter, Struct. Defects Mech. Prop. 1998, 77 (3), 621-640. https://doi.org/10.1080/01418619808224072.

[33] Takeda, M.; Ina, H.; Kobayashi, S. Fourier-Transform Method of Fringe-Pattern Analysis for Computer-Based Topography and Interferometry. J. Opt. Soc. Am. 1982, 72 (1), 156. https://doi.org/10.1364/josa.72.000156.

[34] Dabuechies, I. The Wavelet Transform, Time-Frequency Localization and Signal Analysis. IEEE Trans. Inf. Theory 1990, 36 (5), 961-1005. https://doi.org/10.1109/18.57199. 
[35] Fu, C. C.; Grimes, A.; Long, M.; Ferri, C. G. L.; Rich, B. D.; Ghosh, S.; Ghosh, S.; Lee, L. P.; Gopinathan, A.; Khine, M. Tunable Nanowrinkles on Shape Memory Polymer Sheets. Adv. Mater. 2009, 21 (44), 4472-4476.

https://doi.org/10.1002/adma.200902294.

[36] Schneider, C. A.; Rasband, W. S.; Eliceiri, K. W. NIH Image to ImageJ: 25 Years of Image Analysis. Nat. Methods 2012, 9 (7), 671-675. https://doi.org/10.1007/978-184882-087-6_9.

[37] Canny, J. A Computational Approach to Edge Detection. IEEE Trans. onf Pattern Anal. Mach. Intell. 1986, 8 (6), 697-698.

https://doi.org/10.1109/ASICON.2011.6157287.

[38] Lim, J. S. Two-Dimensional Signal and Image Processing. Englewood Cliffs, NJ, Prentice Hall, 1990, 710 p. 1990.

[39] Frigo, M.; Johnson, S. G. The Design and Implementation of FFTW3. Proc. IEEE 2005, 93 (2), 216-231. https://doi.org/10.1109/JPROC.2004.840301.

[40] Van Der Schaaf, A.; Van Hateren, J. H. Modelling the Power Spectra of Natural Images: Statistics and Information. Vis. Res 1996, 36 (17), 2759-2770.

[41] Wagner, D.; Novog, D. R.; Lapierre, R. R. Simulation and Optimization of Current Generation in Gallium Phosphide Nanowire Betavoltaic Devices. J. Appl. Phys. 2019, 125 (16). https://doi.org/10.1063/1.5093805.

[42] Bian, G.; Dickey, J. M. Properties of Multivariaate Cauchy and Poly-Cauchy Distributions with Bayesian g-Prior Applications; 1991.

[43] De France, K. J.; Babi, M.; Vapaavuori, J.; Hoare, T.; Moran-Mirabal, J.; Cranston, E. D. 2.5D Hierarchical Structuring of Nanocomposite Hydrogel Films Containing Cellulose Nanocrystals. ACS Appl. Mater. Interfaces 2019, 11 (6), 6325-6335. https://doi.org/10.1021/acsami.8b16232.

[44] Makaremi, S.; Luu, H.; Boyle, J. P.; Zhu, Y.; Cerson, C.; Bowdish, D. M. E.; Moran- 
Mirabal, J. M. The Topography of Silica Films Modulates Primary Macrophage

Morphology and Function. Adv. Mater. Interfaces 2019, 6 (21), 1-9.

https://doi.org/10.1002/admi.201900677.

[45] Guvendiren, M.; Burdick, J. A. The Control of Stem Cell Morphology and Differentiation by Hydrogel Surface Wrinkles. Biomaterials 2010, 31 (25), 6511-6518. https://doi.org/10.1016/j.biomaterials.2010.05.037. 
Synopsis: The topographical characterization of material interfaces is key to determining structure-function relations for a variety of applications including biosensors, flexible electronics, or implant materials. This work presents a step-by-step method for the accurate quantification of structured surface topography that optimizes feature size extraction while reducing user bias and error.

Keywords: Fourier transform; microfabrication; wrinkling; mechanical buckling; image filter; nanomaterials; edge detection; feature size; topographical characterization

ToC figure

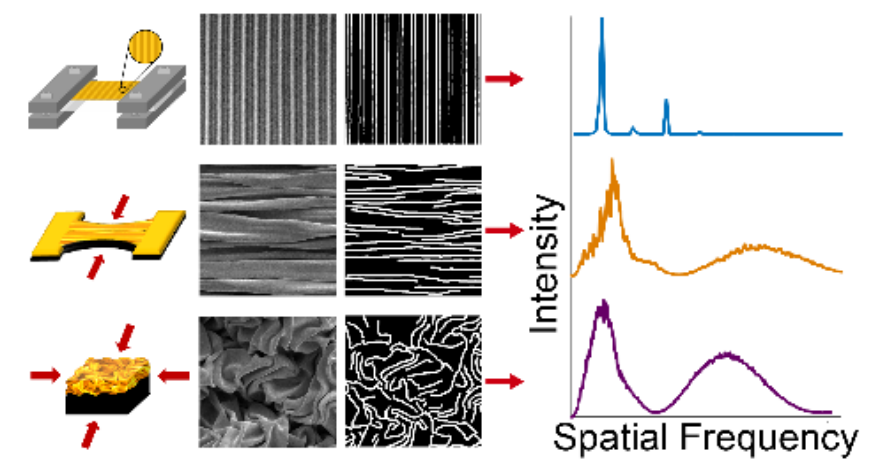

